\title{
Single Crystal Electron Crystallography on Organic Molecules
}

\author{
Ute Kolb*, Galina Matveeva** \\ * Institut für Physikalische Chemie, Johannes Gutenberg Universität, Welderweg 11, 55099 Mainz, \\ Germany. \\ ** Institute of Macromolecular Compounds of the Russian Academy of Sciences, Bolshoi pr. 31, \\ 199004 St. Petersburg, Russia.
}

Many compounds crystallize not only in small crystals non-suitable for single crystal x-ray structure analysis, they also exist in different modifications. The structures of these materials can be accessed by single crystal electron diffraction and $\mathrm{x}$-ray powder measurements.

X-ray powder diffraction delivers two-dimensional data, which suffers often from overlapping reflections, inadequate crystal quality, unknown impurities and preferred orientation. Electron diffraction on the other hand provides us with three-dimensional data. Unfortunately, it suffers from an incomplete reciprocal space (missing cone problem), elongated reflections (spike function) and is effected by multiple scattering and dynamical scattering for thick samples. Because dynamical scattering is less serious for organic materials, structure determination can be carried out based on kinematical procedures in the first step and the resulting model can be refined dynamically in a second step. The combination of both approaches provides us with the tool for structure analysis of organic molecules [1].

The lack of reliable intensities, which $\mathrm{x}$-ray powder diffraction and single crystal electron diffraction have in common, leads often to a failure of approaches, which use only intensity information for an "ab initio" structure analysis such as "direct methods" or Patterson approach. Examples for successful use of the tangent formula or Sayre equation for structure determination from electron diffraction data are worked out intensely by D. Dorset [2]. Other direct methods like maximum entropy can provide us with an envelope of the molecules in the cell, which gives an idea of its orientation [3-5].

To optimize their physical properties which are strongly influenced by the structure we investigated a number of non-linear optical active compounds for laser applications. All crystallize in noncentrosymmetric space groups: 1-(2-furyl),3-(4-benzamidophenyl)-2-propene-1-one (FAPPOBE) has a non-linear optical activity of approx. $100 \mathrm{x}$ urea whereas (E)-1(4 aminophenyl)-3-(4 bromophenyl)2-propen-1-one (U800) shows a non-linear optical activity up to 800 times urea. The third compound 1-(2-furyl),3-(4-aminophenyl)-2-propene-1-one (FAPPO) crystallized in three modifications which could not be purified. The structure analysis for two of the three identified modifications was performed only based on electron diffraction data.

Approx. 100-200 Å thick crystals were grown from hot ethanol or toluene. In diffraction mode three dimensional data sets were measured on a Philips EM300 at 100keV and a FEI TECNAI F30ST by tilting the sample with a rotation-tilt holder around suitable axes (max. tilt angle $\pm 60^{\circ}$ ). Determination of the orthorhombic unit cells (Pna2 $1, \mathrm{Pca}_{1}$ and Fdd2) were performed from approx.15 zones originating from a tilt around main axes. Intensities were collected using an Nikon AF-4500 scanner with 2400 dpi resolution and 12 bit grey level or a 1024x1024 slow-scan CCD. 
Refinement of cell parameters we used x-ray powder diffraction pattern measured in transmission and fitted with a Pawley Fit with DASH or Cerius2 [6, 7].

The conformation of the molecules in the gas phase as well as dipole $\mu$ and hyperpolarisability $\beta$ were calculated by semiempirical, quantummechanical methods (MOPAC6.0 ; PM3)[8]. Alternative approaches are the search of the Cambridge Database [9] or "ab initio" calculations [10].

For simulation methods cell dimensions, space group, the initial model of the molecule and structure factor amplitudes were used. Packing energy minimisation [11] and simulation of diffraction patterns [7] were performed alternatingly gaining a good R-factor for each zone. Based on the obtained atomic coordinates, hyperpolarizability tensors were calculated using quantum mechanical methods and related to the crystal by appropriate co-ordinate transformation [12]. Separately, a 3D-data set was buildt up and statistical ab initio methods such as „Maximum Entropy“ $[13,14]$ were used.

[1]I.G. Voigt-Martin, U. Kolb, International School of Crystallography, $26^{\text {th }}$ course: Electroncrystallography, Erice (1997).

[2] D.L. Dorset, Structural Electron Crystallography, New York: Plenum Press 1995.

[3] G. Bricogne, Acta Cryst. A Found Cryst. A46:830, 1984.

[4] G. Bricogne and C. Gilmore, Acta Cryst. A Found Cryst. A46:284, 1990.

[5] C.J. Gilmore, G. Bricogne and G. Bannister, Acta Cryst. A Found Cryst. A47:830, 1991.

[6] DASH - CCDC Software Ltd., Cambridge, GB.

[7] Cerius2 version 4.2MS, Molecular modeling environment from Accelrys Inc., 9685 Scranton Road, San Diego, CA 92121-3752, USA.

[8] J.J.P. Stewart, MOPAC6.0 A General Purpose Molecular Orbital Package, QCPE.

[9] CSD - Cambridge structural database, CCDC Software Ltd., Cambridge, GB.

[10] TURBOMOL v235, Biosym Technologies, San Diego, 1993.

[11] DREIDING2.21, S.L. Mayo, B.D. Olafson, W.A. Goddard III J. Phys. Chem. 94:8897-8909, 1990.

[12] A.V. Yakimanski et al., Acta Cryst. A53, (1997) 603-614.

[13] I.G. Voigt-Martin, et al. Ultramicroscopy 68, (1997) 43-59.

[14] I.G. Voigt-Martin, et al. Phys. Rev. B59, 10, (1999) 6722-. 